\title{
Dietary black-grained wheat intake improves glycemic control and inflammatory profile in patients with type 2 diabetes: a randomized controlled trial
}

\author{
This article was published in the following Dove Press journal: \\ Therapeutics and Clinical Risk Management
}

\author{
Yanping Liu',* \\ Ju Qiu',* \\ Yanfen Yue $^{3}$ \\ Kang $\mathrm{Li}^{3}$ \\ Guixing $\operatorname{Ren}^{4}$ \\ 'Department of Nutrition, Peking \\ Union Medical College Hospital, \\ Beijing, ${ }^{2}$ Institute of Food and \\ Nutrition Development, Ministry of \\ Agriculture, Beijing, ${ }^{3}$ Department \\ of Nutrition, Pinggu Hospital of \\ Traditional Chinese Medicine, Beijing, \\ ${ }^{4}$ Institute of Crop Sciences, Chinese \\ Academy of Agricultural Sciences, \\ Beijing, China \\ *These authors contributed equally \\ to this work
}

\begin{abstract}
Introduction: Although black-grained wheat (BGW) is recognized as a nutritional food for humans in China, it has yet to be utilized well for industrial applications, which can be attributed to the limited research data available on its health benefits. Thus, the hypothesis was tested that a daily substitution of BGW for a partial staple food would improve glycemia and inflammatory profile of type 2 diabetes mellitus (T2DM) patients by a randomized controlled trial.
\end{abstract}

Materials and methods: A total of 120 patients were randomly divided between control group (diet control and nutritional education) and BGW group (daily substitution of BGW for a partial staple food).

Results: Based on the significant difference between BGW and control groups $(P<0.05)$, the primary outcomes were that BGW treatment in diet resulted in a significant lowering of glycated albumin (GA, 18.05 to $16.06 \mathrm{mmol} / \mathrm{L}$ ) level in T2DM patients after a 5-week intervention, and this treatment regimen was much more efficient than the strategy of diet control alone. In addition, BGW supplementation prevented the increase in tumor necrosis factor (TNF)- $\alpha$ and interleukin (IL)-6 induced by T2DM. There were no significant differences in blood glucose, glycated hemoglobin or insulin levels between the 2 groups. The subgroup analyses of the BGW daily intake showed that, except the TNF- $\alpha$, significant improvements in GA and IL-6 were observed when the BGW intake dose was $>69 \mathrm{~g} /$ day.

Conclusion: These findings support the hypothesis that BGW may improve glycemia and the inflammatory profile in T2DM patients.

Keywords: black-grained wheat, glycated albumin, tumor necrosis factor- $\alpha$, interleukin- 6

\section{Introduction}

Type 2 diabetes mellitus (T2DM) consumes a large portion of health care expenditures and will continue to place a heavy burden on health care budgets in the future. ${ }^{1}$ Medical nutrition therapy has been reported to improve diabetic outcomes, which is highly recommended for the management of T2DM patients. ${ }^{2,3}$ Consumption of whole grains or coarse grains has been associated with a variety of health benefits that are closely associated with T2DM. Although the exact mechanisms have yet to be discovered, it is understood that the bioactive compounds, especially in the bran of cereal grains, play a key role in their physiological functions. ${ }^{4}$

Black-grained wheat (BGW) is a new wheat variety which has been in development in China during the last 20 years; its black-grained genotype was developed from a previously existing blue and purple line. ${ }^{5,6}$ It is now available for utilization
Correspondence: Guixing Ren Institute of Crop Sciences, Chinese Academy of Agricultural Sciences, No 80 Xueyuan South Road, Haidian District, Beijing 100081, China

Tel +86 I06 2II 5596

Fax +861062156596

Email renguixing@caas.cn 
as a new raw food material for value-added products. ${ }^{7-9}$ The nutritional value of $\mathrm{BGW}$ is close to its colored seed coat. The main pigment components of the BMG seed coat are the anthocyanins. ${ }^{10}$ Anthocyanins are naturally occurring polyphenolic compounds abundant in dark-colored fruits and vegetables, and also accumulate in pigmented grains, such as black rice, red sorghum, and purple maize. ${ }^{11,12}$ Epidemiological studies suggest that the increased consumption of anthocyanins lowers the risk of T2DM. ${ }^{12}$ The mechanisms of action of anthocyanins are primarily related to their antioxidant properties. ${ }^{13}$ BMG has been reported to be a potential source of natural antioxidants. ${ }^{14}$ In addition to anthocyanins, the polysaccharide ${ }^{15}$ and protein ${ }^{16}$ are also important nutrients for the utilization of BGW. The contents of polysaccharide and protein of BGW are higher than that found in common wheat, which plays a key role in the BGW bioactivity. Considering these valuable compositions, BGW should receive more attention as an alternative source of nutrients and health-protective components in the human diet.

BGW has been used as raw food material in China, but less is known about its health benefits in the human diet. Its use in the food industry is still limited when compared with other types of grains such as wheat, rye, and oat. Whole wheat and rye grains are common ingredients of the human diet, serving as essential sources of dietary fiber. A rye-based diet enhances early insulin secretion, which lowers the risk of deteriorating glucose tolerance and T2DM development. ${ }^{17,18}$ Oat has also been reported to be effective for improving T2DM. ${ }^{19}$ Because of the presence of many bioactive compounds, BGW may be the potential resource of nutraceutical and functional materials for the development of anti-diabetic food. However, we are not aware of any research related to the anti-diabetic effect of BGW, neither animal experiment nor human experiment. Therefore, with a 5-week intervention, we tested the hypothesis that daily substitution of BGW for a partial staple food will improve T2DM risk factors, and the changes in fasting glucose, glycated hemoglobin ( $\mathrm{HbAlc})$, insulin resistance and inflammatory profile of T2DM subjects were assessed.

\section{Materials and methods Subjects}

Subject screening was conducted at Pinggu Hospital of Traditional Chinese Medicine (Beijing, China) based on the annual physical examination results (collected in 2016) of patients assessed in the past. Patients with T2DM between 30 and 80 years of age, who were diagnosed as diabetic or used hypoglycemic agents, were invited to take part in the present study. Subjects were eligible if they presented the following components: 1) fasting glucose $>6.1 \mathrm{mmol} / \mathrm{L}$; 2) $\mathrm{HbA} 1 \mathrm{c}>6.5 \%$; 3) previously diagnosed as diabetic; or 4) using hypoglycemic agents. Subjects were excluded if they had a history of severe kidney disease, cancer or psychological disorders, and pregnant or lactating women were excluded as well. The sample size was calculated according to a study of oat-induced improvement in fasting glucose in Chinese T2DM patients. ${ }^{19}$ The minimum sample size was 60 subjects per group with a confidence level of $95 \%$ and a power of $90 \%$. To recruit 134 subjects, considering a $10 \%$ dropout rate, a total of 143 subjects attended the invitation and a subsequent biunique screening. After the exclusion of subjects ineligible to take part in the study, the remaining subjects were divided into random groups using a random number table method with SPSS for WINDOWS 19.0 (SPSS Inc., Chicago, IL, USA). Finally, 120 subjects were screened and randomly assigned to a diet control group (control group) or BGW food intervention group (BGW group; Figure 1).

\section{Study design}

This study was a parallel, randomized, open-label controlled trial to compare the influences of BGW with refined wheat or rice (control) on markers of metabolic syndrome (MetS) in T2DM subjects during a 5-week intervention test period (Trial registration: NCT02999867 at ClinicalTrials.gov). The study protocol was approved by The Ethics Committee of Peking Union Medical College Hospital (ZS-1048) and was consistent with both the Declaration of Helsinki of 1975, as revised in 1983, and the guidelines of the center's institutional review board. A written informed consent was obtained from each patient. All subjects received intensive nutritional education, mainly related to dietary nutrition and T2DM management every week during follow-up in the test period. Systematic dietary programs were designed according to the Dietary Guidelines for the Chinese Resident 2013 and the China Medical Nutrition Therapy Guideline for Diabetes 2010. ${ }^{19}$ Based on the information of individual energy and nutritional requirements for subjects calculated from their physical examination results at baseline, the recommended recipes were offered to each patient, which provided details of the requirements of daily calorie and dietary intake of different varieties of food. The control group was assigned to consume wheat or rice as daily cereals, while the BGW group was assigned to replace a part of rice and wheat with equivalent 


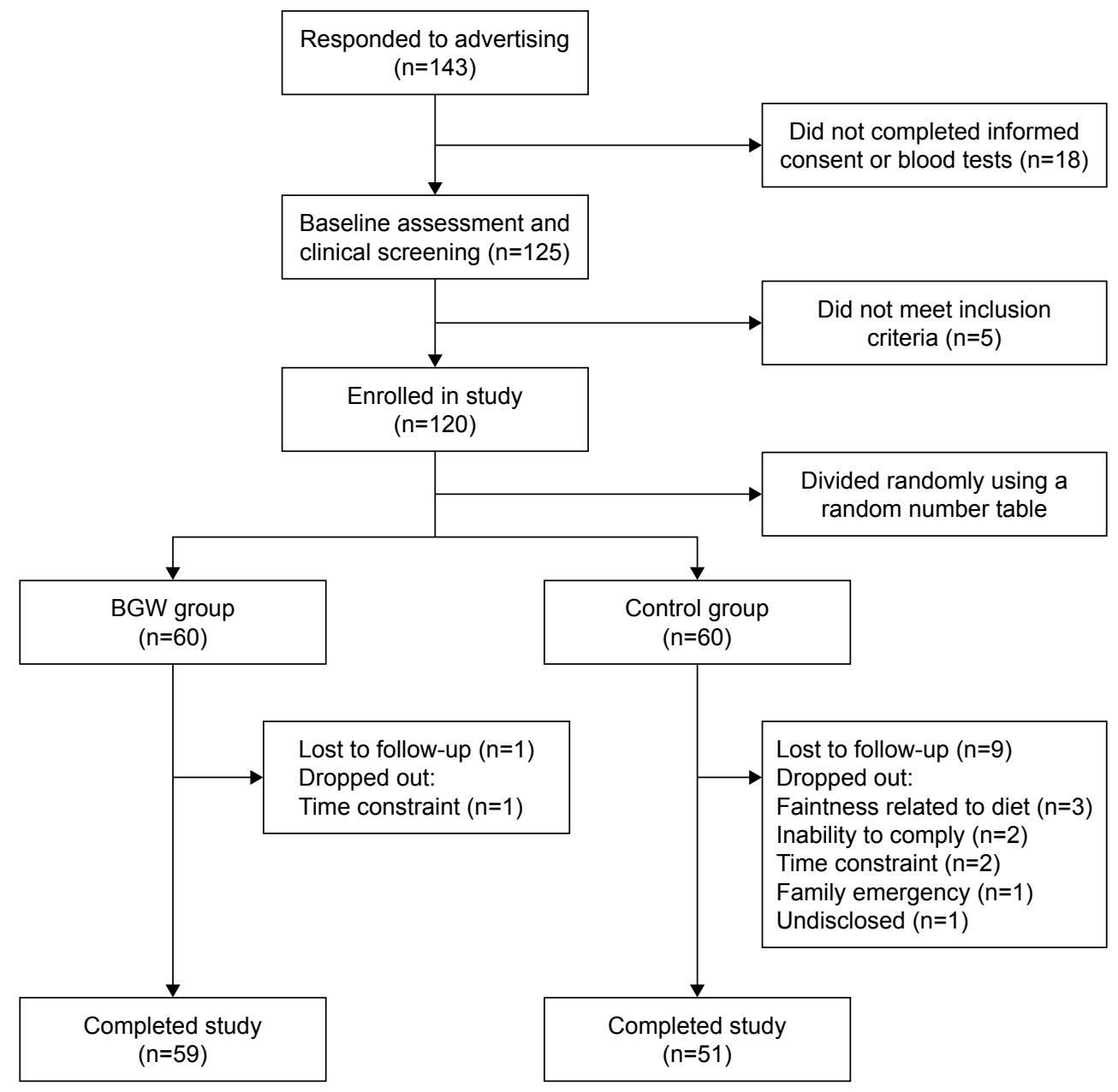

Figure I Flow diagram of the study.

Abbreviation: BGW, black-grained wheat.

BGW foods. BGW foods (steamed bun, noodles and kernel) were wrapped in a package ( $50 \mathrm{~g}$ per serving) and provided to the subjects, and the exact intake of cereals or other foods was recorded in a diet diary. All subjects were required to maintain their habits of physical activity and medication during the test period.

Compliance was monitored by researchers who weighed the uneaten cereal packets returned by the subjects each week. Together with the daily records of food intake in the formulary diet diary, including quantity and frequency of test cereals and other foods eaten during the study, compliance with the intervention was determined. Although it was not possible for subjects to be unaware of their assignment due to the obvious differences in appearance and texture between BGW foods and wheat or rice, the technicians or statisticians responsible for the preliminary evaluation of the outcomes were blind to the allocation, because the grouping methods and the patient's dietary design were not known to them.

\section{Supplement characteristics}

BGW foods, including the steamed bun, noodles and kernels, were developed by Institute of Crop Sciences, Chinese Academy of Agricultural Sciences. BGW (local variety), harvested in autumn 2016 in Hebei Province of China, was peeled and milled to flour for further processing. The steamed bun was made of mixed flour of BGW (74\%), mung bean and glutenin, followed by kneading dough with yeast and water (the mixed flour: water ratio was 1:0.5 by weight). The noodles were made of mixed flour of BGW (72\%), mung bean, konjac and glutenin, followed by rolling cutting and drying. The kernels were made of peeled BGW (24\%) that was mixed with mung bean and rice. Subjects cooked BGW foods for meals according to standard protocols as follows: steamed buns were steamed for $15 \mathrm{~min}$; BGW noodles were boiled in water for $10 \mathrm{~min}$; kernels were steamed with water (the kernel: water ratio was $1: 2$ by weight) in a rice cooker for $25 \mathrm{~min}$. The nutrient contents of these $3 \mathrm{BGW}$ foods 
Table I Nutritional composition of the BGW test foods

\begin{tabular}{|c|c|c|c|c|c|c|}
\hline Food types & $\begin{array}{l}\text { Energy, } \\
\text { kcal// } 00 \text { g }\end{array}$ & $\begin{array}{l}\text { Protein, } \\
\mathrm{g} / 100 \mathrm{~g}\end{array}$ & $\begin{array}{l}\text { Fat, } \\
\text { g/l00 g }\end{array}$ & $\begin{array}{l}\text { Carbohydrate, } \\
\mathrm{g} / 100 \mathrm{~g}\end{array}$ & $\begin{array}{l}\text { Dietary fiber, } \\
\mathrm{g} / 100 \mathrm{~g}\end{array}$ & $\begin{array}{l}\text { BGW, } \\
\text { g/100 g }\end{array}$ \\
\hline Steamed bun & 965 & 16.6 & 1.6 & 37.2 & 4.9 & 49.6 \\
\hline Noodles & $\mathrm{I}, 409$ & 19.4 & 1.8 & 54.9 & 10.0 & 72 \\
\hline Kernels & $\mathrm{I}, 448$ & II.I & I.I & 71.4 & 2.7 & 24 \\
\hline
\end{tabular}

Note: Data are expressed as the means $\pm \operatorname{SD}(n=3)$.

Abbreviation: BGW, black-grained wheat.

(Table 1) were determined using standardized analytical methods, including the Association of Official Analytical Chemists International method.

\section{Dietary assessment}

Dietary intake was assessed by 3-day food records, including 1 weekend day. After a biunique direction by trained workers, subjects recorded their own daily food intake using the formulary diet diary every day, which was then summarized by trained investigators each week. The intake data were analyzed by using the nutrition clinic consultation management system (Zhending Health Technology Co. Ltd., Shanghai, China).

\section{Measurements}

Physical examinations were performed by trained nurses at baseline and the end of the 5-week intervention period. Body weight, height, body mass index, systolic and diastolic blood pressures were detected according to standard methods. ${ }^{20}$ Blood samples were drawn once from subjects in the morning after an overnight fast. Plasma glucose, HbAlc, serum insulin, and the homeostasis model of assessment insulin resistance (HOMA-IR) were measured according to our pervious methods. ${ }^{21}$ Serum glycated albumin (GA) was measured using the LUCICA GA-L kit (Asahi Kasei Pharma Corporation, Tokyo, Japan) and analyzed by the automated ADVIA 1650 instrument (Siemens Medical Solutions Diagnostics, Tarrytown, NY, USA). Plasma tumor necrosis factor- $\alpha$ (TNF- $\alpha$ ) and interleukin-6 (IL-6) were measured using the enzyme-linked immunosorbent assay technique from R\&D Systems (Minneapolis, MN, USA). ${ }^{22}$ Hypersensitive-C reactive protein (hs-CRP) was determined by a high-sensitivity assay using a BN II nephelometer (Dade Behring, Marburg, Germany). ${ }^{23}$

\section{Statistical analyses}

Data were expressed as mean $\pm \mathrm{SD}$ or median (interquartile range) for continuous variables and as number (percentage) for categorical variables. Variables of HOMA-IR, TNF- $\alpha$, and IL- 6 were transformed by the natural logarithm because their distributions were heavily skewed. The statistical analyses were performed according to our previous methods. ${ }^{21}$ Baseline characteristics of the 2 groups were compared using the Student's $t$-test, Wilcoxon's signed-rank test, or a Pearson's $\chi^{2}$ test as appropriate. Differences in variables between the groups at baseline or after the 5-week intervention were analyzed using the Student's $t$-test, and differences between the baseline and final variable measurements after the 5-week intervention were compared using the paired Student's $t$-test. The changes in the glycemic control, inflammatory profiles, and dietary intake from baseline were analyzed using an analysis of covariance (ANCOVA, generalized linear model). All data were analyzed with an intention-to-treat basis without imputation using SPSS software for WINDOWS 19.0 (SPSS Inc, Chicago, IL, USA) and a 2 -sided $P<0.05$ was considered significant.

\section{Results}

\section{Baseline characteristics}

After randomization, a total of 120 subjects obtained fairly balanced diet control (Figure 1). One hundred and ten subjects were considered capable of completing the 5-week intervention. No significant difference in major characteristics of subjects between the 2 groups was observed at baseline $(P>0.05)$ (Table 2).

\section{Dietary intake}

Daily energy and nutrient intake was analyzed (Table 3) based on the nutritional composition of the BGW foods (Table 1) and the daily diary obtained from the subjects. No differences were found in the level of energy at 5 weeks and the change of energy from baseline between BGW and control groups, although the energy at baseline was different. After the 5-week intervention, the BGW treatment group showed a higher intake of protein $(P=0.012)$ and dietary fiber $(P<0.001)$ than the control group. The changes in protein $(P=0.006)$ and dietary fiber $(P<0.001)$ intake were also significantly different between the 2 groups. In addition, the paired $t$-test analysis showed that protein intake increased significantly after the 5-week intervention in the 
Table 2 Characteristics of the subjects at baseline

\begin{tabular}{|c|c|c|c|}
\hline Variable & $\begin{array}{l}\text { BGW } \\
\text { group } \\
(n=59)\end{array}$ & $\begin{array}{l}\text { Control } \\
\text { group } \\
(n=5 I)\end{array}$ & $P$-value \\
\hline Male, n (\%) & $26(44.1)$ & $24(47.1)$ & 0.444 \\
\hline Age (years) & $58.5 \pm 10.2$ & $57.4 \pm 8.8$ & 0.544 \\
\hline Duration of diabetes (years) & $7(0.3-20)$ & $9(0.3-20)$ & 0.102 \\
\hline Family history of diabetes, $\mathrm{n}(\%)$ & $26(44.1)$ & $26(51.0)$ & 0.469 \\
\hline \multicolumn{4}{|l|}{ Diabetes medication use } \\
\hline No diabetes medication, $\mathrm{n}(\%)$ & $19(32.2)$ & $20(39.2)$ & 0.443 \\
\hline Oral medication only, n (\%) & $38(64.4)$ & $37(72.5)$ & 0.361 \\
\hline Insulin only, n (\%) & $5(8.5)$ & $2(3.9)$ & 0.329 \\
\hline Oral medication and insulin, $\mathrm{n}(\%)$ & $7(11.9)$ & $6(11.8)$ & 0.987 \\
\hline Antihypertensive agents & $14(23.7)$ & $9(17.6)$ & 0.434 \\
\hline Lipid-lowering agents & $3(5.1)$ & $6(11.8)$ & 0.202 \\
\hline Complications, n (\%) & $16(27.2)$ & $12(23.5)$ & 0.667 \\
\hline MetS (NCEP ATP III), n (\%) & $31(52.5)$ & $23(45.1)$ & 0.452 \\
\hline Overweight, n (\%) & $49(83.1)$ & $37(72.5)$ & 0.248 \\
\hline Hypertension, n (\%) & $29(49.2)$ & $2 I(4 I .2)$ & 0.446 \\
\hline Dyslipidemia, n (\%) & $46(78.0)$ & $36(70.6)$ & 0.390 \\
\hline Weight, kg & $70.6 \pm 12.3$ & $68.1 \pm 10.7$ & 0.273 \\
\hline Height, cm & $161.9 \pm 7.6$ & $161.4 \pm 8.4$ & 0.914 \\
\hline $\mathrm{BMI}, \mathrm{kg} / \mathrm{m}^{2}$ & $26.8 \pm 3.4$ & $26.1 \pm 3.2$ & $0.28 \mathrm{I}$ \\
\hline Systolic blood pressure, $\mathrm{mmHg}$ & $|4| . \mid \pm 17.4$ & $136.6 \pm 17.7$ & 0.181 \\
\hline Diastolic blood pressure, $\mathrm{mmHg}$ & $79.4 \pm 11.9$ & $76.9 \pm 10.2$ & $0.24 I$ \\
\hline
\end{tabular}

Notes: Data are expressed as the means \pm SD for normally distributed variables, including age, weight, height and blood pressure; medians (IQR) for non-normally distributed variables, including duration of diabetes; or number (\%) for categorical variables. Proportions were compared using Pearson's $\chi^{2}$ test, the means were compared using Student's $t$-test, and medians were compared using the Wilcoxon's signed-rank test. Overweight is defined as BMI $\geq 24 \mathrm{~kg} / \mathrm{m}^{2}$. Hypertension is defined as elevated blood pressure, having been diagnosed as hypertensive, or using antihypertensive agents. Dyslipidemia is defined as $L D L$ cholesterol $\geq 3.3 \mathrm{mmol} / \mathrm{L}$, reduced HDL cholesterol or elevated TG, having been diagnosed as dyslipidemic, or using lipid-lowering agents.

Abbreviations: BGW, black-grained wheat; BMI, body mass index; IQR, interquartile range; MetS, metabolic syndrome; NCEP ATP III, National Cholesterol Education Program Adult Treatment Panel III; TG, triglycerides; HDL, high-density lipoprotein; LDL, low-density lipoprotein.

BGW group $(P<0.001)$, but not control group; the dietary fiber intake increased in the BGW group $(P<0.001)$ but decreased in the control group $(P<0.05)$. Besides, the energy obtained from protein by the $\mathrm{BGW}$ group was significantly higher than the control group $(P<0.001)$, while the energy obtained from carbohydrate by the BGW group was lower $(P<0.001)$. The changes in levels of energy obtained from protein $(P<0.001)$ and carbohydrate $(P=0.006)$ were also significantly different between the 2 groups. Compared with baseline, the energy obtained from protein was increased by BGW treatment $(P<0.001)$, but energy obtained from carbohydrate was decreased $(P<0.001)$; simultaneously, these were not affected in the control group.

\section{Glycemic control and insulin sensitivity}

The GA level of BGW group was not different from that of the control group at baseline $(P=0.524)$, but it was significantly different between the 2 groups after the 5 -week intervention ( $P=0.034$ ) (Table 4). Although the GA levels of the BGW and control groups were both decreased significantly based on the paired Student's $t$-test analysis $(P<0.05)$, the changes in GA level from baseline was significantly different between the 2 groups $(P=0.002)$. No significant differences were found in plasma glucose and $\mathrm{HbA} 1 \mathrm{c}$ between the $\mathrm{BGW}$ and control groups $(P>0.05)$. Insulin and HOMA-IR were normalized using logarithmic transformations. As shown in Table 4, neither the logarithm of insulin nor that of HOMA-IR was affected significantly by BGW treatment $(P>0.05)$.

\section{Inflammatory profile}

The TNF- $\alpha$ level of the BGW group was not different from that of the control group at baseline $(P=0.116)$, but it was significantly different between the 2 groups after the 5-week intervention $(P=0.003)$ (Table 4). The change in TNF- $\alpha$ from baseline was also significantly different between the 2 groups $(P=0.001)$. Based on the paired Student's $t$-test analysis, TNF- $\alpha$ level was found to be significantly increased after the 5-week intervention in the control group $(P<0.05)$, but no significant change in TNF- $\alpha$ was observed in the BGW group $(P>0.05)$. The change in IL-6 level from baseline between groups tended to be different $(P=0.081)$. Although no difference was found in IL-6 level at 5 weeks because of the lower level of IL-6 in the control group compared with the BGW group at baseline, the IL-6 level was increased in the control group $(P<0.05)$, but not the $\mathrm{BGW}$ group $(P>0.05)$, during the test period. Compared with the significant increases in inflammatory biomarkers of the control group, BGW treatment balanced the levels of both TNF- $\alpha$ and IL- 6 . No significant differences were found in the hs-CRP level between the BGW and control groups $(P>0.05)$.

\section{Subgroup analyses}

Subgroups were divided into 2 subgroups according to the different daily intake of BGW (69 $\mathrm{g} / \mathrm{d}$ as a median of daily intake) in order to investigate the possible effective dose of BGW. The glycemic control and inflammatory profiles among the various $\mathrm{BGW}$ intake groups were reanalyzed using ANCOVA with post hoc analyses (Table 5). There were 29 subjects having an average BGW dose of $<69 \mathrm{~g} / \mathrm{d}$ in the low BGW group and 30 subjects having $>69 \mathrm{~g} / \mathrm{d}$ in the high BGW group. Subjects in the high BGW group showed a significantly lower GA concentration compared with the no BGW group $(P<0.05)$, while the GA concentration of low $\mathrm{BGW}$ group was not different from the no $\mathrm{BGW}$ group. A significant decrease in IL-6 level was also discovered in 
Table 3 Daily energy and nutrient intake of the subjects at baseline and after a 5-week dietary intervention

\begin{tabular}{|c|c|c|c|}
\hline Variable & BGW group $(n=59)$ & Control group $(n=5 I)$ & $P$-value \\
\hline \multicolumn{4}{|l|}{ Energy, kcal } \\
\hline Baseline & $\mathrm{I}, 834.16(\mathrm{I}, 742.43$ to $\mathrm{I}, 925.89)$ & $2,032.43(I, 924.67$ to $2,140.19)$ & 0.034 \\
\hline 5-weeks & $\mathrm{I}, 735.33(\mathrm{I}, 654.23$ to $\mathrm{I}, 8 \mathrm{I} \mid 6.43)$ & $\mathrm{I}, 854.10(\mathrm{I}, 756.7 \mathrm{I}$ to $\mathrm{I}, 95 \mathrm{I} .49)$ & 0.154 \\
\hline Change from baseline & $-98.83(-189.13$ to -8.53$)$ & $-178.33(-291.76$ to -64.90$)$ & 0.404 \\
\hline \multicolumn{4}{|l|}{ Protein, $g$} \\
\hline Baseline & 37.72 (33.06 to 42.38$)$ & 38.97 (34.19 to 43.75$)$ & 0.594 \\
\hline 5-weeks & $45.04(40.10 \text { to } 49.98)^{a}$ & 39.28 (35.08 to 43.48$)$ & 0.012 \\
\hline Change from baseline & $7.32(2.22$ to 12.42$)$ & 0.31 ( -4.66 to 5.28$)$ & 0.006 \\
\hline \multicolumn{4}{|l|}{ Protein, \% of energy } \\
\hline Baseline & I2.25 (11.43 to 13.07) & 11.38 (10.65 to 12.12) & 0.052 \\
\hline 5-weeks & I5.80 (I5.09 to I6.5I $)^{\mathrm{a}}$ & 12.55 (II.83 to 13.27) & $<0.001$ \\
\hline Change from baseline & $3.55(2.5$ I to 4.59$)$ & $1.17(0.19$ to 2.15$)$ & $<0.001$ \\
\hline \multicolumn{4}{|l|}{ Fat, g } \\
\hline Baseline & $57.30(53.13$ to 61.47$)$ & 62.65 (57.34 to 67.96$)$ & 0.253 \\
\hline 5-weeks & $5 \mathrm{I} .92(49.23$ to $54.6 \mathrm{I})$ & 54.34 (50.86 to 57.82$)$ & 0.443 \\
\hline Change from baseline & $-5.38(-9.32$ to -1.44$)$ & -8.31 ( -13.73 to -2.89$)$ & 0.517 \\
\hline \multicolumn{4}{|l|}{ Fat, \% of energy } \\
\hline Baseline & $19.00(17.64$ to 20.36$)$ & $18.23(17.05$ to 19.41$)$ & 0.531 \\
\hline 5-weeks & $18.48(17.62$ to 19.34$)$ & I7.42 (I6.53 to I8.3I) & 0.227 \\
\hline Change from baseline & -0.51 ( $(-1.68$ to 0.66$)$ & $-0.82(-1.96$ to 0.34$)$ & 0.815 \\
\hline \multicolumn{4}{|l|}{ Carbohydrate, g } \\
\hline Baseline & 211.31 (194.52 to 228.09$)$ & 239.27 (223.73 to $254.8 \mathrm{I})$ & 0.016 \\
\hline 5-weeks & I83.83 (I7|.28 to |96.38) & 220.97 (204.36 to 237.58$)$ & 0.001 \\
\hline Change from baseline & $-27.48(-43.09$ to -11.88$)$ & $-\mid 8.30(-35.5 \mid$ to -1.08$)$ & 0.431 \\
\hline \multicolumn{4}{|l|}{ Carbohydrate, \% of energy } \\
\hline Baseline & 68.63 (66.84 to 70.43$)$ & 70.44 (68.83 to 72.04$)$ & 0.134 \\
\hline 5-weeks & $64.42(63.32 \text { to } 65.5 \mathrm{I})^{\mathrm{a}}$ & 69.80 (68.38 to 71.22$)$ & $<0.001$ \\
\hline Change from baseline & $-4.22(-5.99$ to -2.44$)$ & $-0.64(-2.47$ to 1.20$)$ & 0.006 \\
\hline \multicolumn{4}{|l|}{ Dietary fiber, $g$} \\
\hline Baseline & 11.93 (10.50 to 13.37) & 13.26 (11.4 to 15.12) & 0.263 \\
\hline 5-weeks & $20.49(19.10 \text { to } 21.88)^{\mathrm{a}}$ & $11.23(10.12 \text { to } 12.33)^{a}$ & $<0.001$ \\
\hline Change from baseline & 8.55 (7.10 to 10.00$)$ & $-2.03(-3.89$ to -0.18$)$ & $<0.001$ \\
\hline \multicolumn{4}{|l|}{ Cholesterol, mg } \\
\hline Baseline & $3 \mid 3.07$ (254.44 to $37|.7|)$ & 261.78 (217.64 to 305.92$)$ & 0.159 \\
\hline 5-weeks & 347.90 (319.07 to 376.72$)$ & $328.99(296.59$ to 361.38$)$ & 0.386 \\
\hline Change from baseline & $34.82(-26.54$ to 96.18$)$ & 67.21 (20.16 to II4.26) & 0.397 \\
\hline \multicolumn{4}{|l|}{ Fatty acids, g } \\
\hline Baseline & 41.83 (37.66 to 45.99$)$ & 45.73 (40.42 to 51.04$)$ & 0.253 \\
\hline 5-weeks & 36.57 (33.88 to 39.26$)$ & 38.28 (34.8I to $4 I .74)$ & 0.443 \\
\hline Change from baseline & $-5.26(-9.20$ to -1.31$)$ & $-7.46(-12.88$ to -2.03$)$ & 0.517 \\
\hline
\end{tabular}

Notes: Baseline and 5-weeks data are expressed as means \pm SD. Differences of variables between groups are analyzed using the Student's $t$-test. Changes from baseline are expressed as means $(95 \% \mathrm{Cl})$ adjusted for sex, age, and $\mathrm{BMI}$ at baseline, and baseline values of relevant outcome variables. Differences of variables between groups are analyzed using the analysis of covariance (ANCOVA, generalized linear model) test. ${ }^{\mathrm{a} D i f f e r e n c e ~ i s ~ c o n s i d e r e d ~ t o ~ b e ~ s i g n i f i c a n t ~ a t ~} P<0.05$; within-group differences (baseline vs 5-weeks) are compared using the paired Student's $t$-test if the between-group differences are significant.

Abbreviations: BGW, black-grained wheat; BMI, body mass index.

the high BGW group $(P<0.05)$, but not in the low BGW group $(P>0.05)$, although there was no significant difference between the low and high BGW groups. Both low and high BGW groups showed decreased TNF- $\alpha$ concentration $(P<0.05)$.

\section{Discussion}

The staple foods of T2DM subjects were partially replaced with the whole grain of BGW, which showed a GA lowering effect, and a TNF- $\alpha$ and IL- 6 attenuating effect during a 5-weeks randomized controlled trial, thus supporting our hypothesis. BGW has been reported to be rich in effective components including anthocyanins, ${ }^{10}$ polysaccharides and proteins, ${ }^{15,16}$ which could positively impact the T2DM outcomes. However, only few reports are available on the bioactivities of $\mathrm{BGW}$, and almost no data are available on its consumption as a whole food in human diet.

The significant differences between the 2 groups showed that, when the changes in levels of energy were the same between the 2 groups, nutrients of diet were improved by the 
Table 4 Changes in metabolic parameters during the study period

\begin{tabular}{|c|c|c|c|}
\hline Variable & BGW group $(n=59)$ & Control group $(n=5 I)$ & $P$-value \\
\hline \multicolumn{4}{|l|}{ Glucose, $\mathrm{mmol} / \mathrm{L}$} \\
\hline Baseline & 8.75 (8.16 to 9.34$)$ & 8.85 (8.10 to 9.60$)$ & 0.789 \\
\hline 5-weeks & 8.30 (7.86 to 8.74$)$ & 8.29 (7.59 to 8.98$)$ & 0.935 \\
\hline Change from baseline ${ }^{b}$ & $-0.4 \mathrm{I}(-0.78$ to 0.04$)$ & $-0.57(-0.96$ to -0.18$)$ & 0.557 \\
\hline \multicolumn{4}{|l|}{$\mathrm{HbAlc} \%$} \\
\hline Baseline & 7.13 (6.82 to 7.44$)$ & 7.08 (6.70 to 7.47$)$ & 0.894 \\
\hline 5-weeks & 6.79 (6.50 to 7.08$)$ & $6.90(6.50$ to 7.30$)$ & 0.623 \\
\hline Change from baseline ${ }^{b}$ & $-0.33(-0.62$ to 0.04$)$ & $-0.20(-0.5$ I to 0.12$)$ & 0.537 \\
\hline \multicolumn{4}{|l|}{$\mathrm{GA}, \mathrm{mmol} / \mathrm{L}$} \\
\hline Baseline & 18.05 (17.0| to 19.09) & 18.54 (17.10 to 19.98) & 0.524 \\
\hline 5-weeks & $16.06(15.37 \text { to } 16.74)^{\mathrm{a}}$ & $17.52(\mid 6.26 \text { to } 18.78)^{a}$ & 0.034 \\
\hline Change from baseline ${ }^{\mathrm{b}}$ & $-2.00(-2.43$ to -1.56$)$ & $-0.95(-1.42$ to -0.48$)$ & 0.002 \\
\hline \multicolumn{4}{|l|}{ Insulin, mU/L } \\
\hline Baseline & $13.32(10.89$ to 15.76$)$ & I2.83 (9.30 to 16.36) & 0.792 \\
\hline 5-weeks & $12.10(10.45$ to 13.75$)$ & II.7| (8.69 to |4.74) & 0.807 \\
\hline Change from baseline ${ }^{b}$ & $-1.13(-2.69$ to 0.43$)$ & $-1.27(-2.95$ to $0.4 I)$ & 0.904 \\
\hline \multicolumn{4}{|l|}{ HOMA-IR, $\mu U \times \mathrm{mol} / \mathrm{L}^{2}$} \\
\hline Baseline & 1.54 (I.39 to I.69) & $1.36(1.24$ to 1.48$)$ & 0.535 \\
\hline 5-weeks & $1.43(1.30$ to 1.56$)$ & $1.34(1.19$ to 1.49$)$ & 0.135 \\
\hline Change from baseline ${ }^{b}$ & $-0.06(-0.18$ to -0.06$)$ & $-0.15(-0.28$ to -0.02$)$ & 0.303 \\
\hline \multicolumn{4}{|l|}{ hs-CRP, mg/L } \\
\hline Baseline & 2.24 (I.4I to 3.06$)$ & 1.63 (0.99 to 2.27$)$ & 0.270 \\
\hline 5-weeks & 2.23 (1.00 to 3.46$)$ & $1.34(0.89$ to 1.80$)$ & 0.212 \\
\hline Change from baseline ${ }^{b}$ & $0.08(-0.77$ to 0.94$)$ & $-0.4 \mid(-1.33$ to 0.52$)$ & 0.448 \\
\hline \multicolumn{4}{|l|}{ TNF- $\alpha, p g / m L$} \\
\hline Baseline & 2.01 ( 1.93 to 2.09$)$ & 1.94 (1.88 to 2.00$)$ & 0.198 \\
\hline 5-weeks & 2.01 (1.92 to 2.10$)$ & $2.36(2.21 \text { to } 2.52)^{\mathrm{a}}$ & 0.003 \\
\hline Change from baseline ${ }^{b}$ & $0.01(-0.10$ to 0.12$)$ & $0.4 \mathrm{I}(0.29$ to 0.53$)$ & 0.001 \\
\hline \multicolumn{4}{|l|}{$\mathrm{IL}-6, \mathrm{pg} / \mathrm{mL}$} \\
\hline Baseline & 0.95 (0.79 to I.10) & 0.74 (0.70 to 0.78$)$ & 0.017 \\
\hline 5-weeks & 0.99 (0.84 to I.I3) & $0.98(0.85 \text { to I.I I })^{\mathrm{a}}$ & 0.957 \\
\hline Change from baseline ${ }^{b}$ & $0.06(-0.05$ to 0.17$)$ & 0.21 (0.09 to 0.33$)$ & 0.081 \\
\hline
\end{tabular}

Notes: Glucose, HbAlc, GA, insulin, and hs-CRP are normally distributed variables. The variables of HOMA-IR, TNF- $\alpha$, and IL- 6 are transformed by the natural logarithm. Baseline and 5 -weeks data are expressed as the means $(95 \% \mathrm{Cl})$. Differences of variables between groups are analyzed using the Student's $t$-test. a Difference is considered to be significant at $P<0.05$; within-group differences (baseline vs 5 -weeks) are compared using the paired Student's $t$-test if the between-group differences are significant. ${ }^{b}$ Changes from baseline of glucose and inflammatory profiles are expressed as means $(95 \% \mathrm{Cl})$ adjusted for sex, age, and $\mathrm{BMI}$ at baseline, and baseline values of the relevant outcome variables. Differences of variables between groups are analyzed using the ANCOVA (generalized linear model) test.

Abbreviations: ANCOVA, analysis of covariance; BGW, black-grained wheat; GA, glycated albumin; HbAIc, glycated hemoglobin; HOMA-IR, homeostasis model assessment of insulin resistance; hs-CRP, hypersensitive-C-reactive protein; IL-6, interleukin-6; TNF- $\alpha$, tumor necrosis factor- $\alpha$.

Table 5 Effect of BGW intake on changes in metabolic parameters during the study period

\begin{tabular}{|c|c|c|c|}
\hline Variable & No BGW (n=5I) & Low BGW (n=29) & High BGW $(n=30)$ \\
\hline Glucose, $\mathrm{mmol} / \mathrm{L}$ & $-0.57(-0.96$ to -0.18$)$ & $-0.43(-1.00$ to 0.14$)$ & $-0.40(-0.99$ to 0.20$)$ \\
\hline $\mathrm{HbAlc}, \%$ & $-0.20(-0.5 \mathrm{I}$ to 0.12$)$ & $-0.25(-0.65$ to 0.14$)$ & $-0.42(-0.97$ to 0.12$)$ \\
\hline $\mathrm{GA}, \mathrm{mmol} / \mathrm{L}$ & $-0.95(-1.42 \text { to }-0.48)^{\mathrm{a}}$ & $-1.20(-1.80 \text { to }-0.60)^{\mathrm{a}}$ & $-2.65(-3.65 \text { to }-1.66)^{b}$ \\
\hline Insulin, mU/L & $-1.27(-2.95$ to $0.4 \mathrm{I})$ & $-0.98(-3.26$ to 1.29$)$ & $-1.53(-3.96$ to 0.89$)$ \\
\hline HOMA-IR, $\mu U \times \mathrm{mol} / \mathrm{L}^{2}$ & $-0.15(-0.28$ to -0.02$)$ & $-0.02(-0.21$ to 0.18$)$ & $-0.12(-0.29$ to 0.05$)$ \\
\hline hs-CRP, mg/L & $-0.4 \mathrm{I}(-1.33$ to 0.52$)$ & $-0.40(-1.22$ to 0.43$)$ & 0.36 (-1.88 to 2.59$)$ \\
\hline TNF- $\alpha, p g / m L$ & $0.4 \mathrm{I}(0.29 \text { to } 0.53)^{\mathrm{a}}$ & $-0.04(-0.16 \text { to } 0.08)^{b}$ & $0.04(-0.05 \text { to } 0.13)^{\mathrm{b}}$ \\
\hline IL-6, pg/mL & $0.21(0.09 \text { to } 0.33)^{a}$ & $0.1 \mathrm{I}(-0.06 \text { to } 0.28)^{\mathrm{a}, \mathrm{b}}$ & $-0.03(-0.18 \text { to } 0.12)^{b}$ \\
\hline
\end{tabular}

Notes: Glucose, HbAlc, GA, insulin, and hs-CRP are normally distributed variables. The variables of HOMA-IR, TNF- $\alpha$, and IL-6 are transformed by the natural logarithm. No BGW, subjects do not eat BGW; Low BGW, intake of BGW is from 30 to $69 \mathrm{~g} / \mathrm{d}$; High BGW, intake of BGW is from 69 to $140 \mathrm{~g} / \mathrm{d}$; $69 \mathrm{~g} / \mathrm{d}$ is the median of BGW intake in the BGW group. All data are mean $(95 \% \mathrm{Cl})$ adjusted for sex, age, $\mathrm{BMI}$ at baseline, and baseline values of the relevant outcome variables. Differences of change from baseline between groups are analyzed using the analysis of covariance (ANCOVAs, generalized linear model) test with post hoc analysis. Different superscript letters represent the statistical differences at $P<0.05$ among the groups.

Abbreviations: ANCOVA, analysis of covariance; BGW, black-grained wheat; GA, glycated albumin; HbA Ic, glycated hemoglobin; HOMA-IR, homeostasis model assessment of insulin resistance; hs-CRP, hypersensitive C-reactive protein; IL-6, interleukin-6; TNF- $\alpha$, tumor necrosis factor- $\alpha$. 
BGW intervention. The replacement of a portion of staple food with BGW increased the energy obtained from protein in diet but decreased the energy obtained from carbohydrate. The BGW supplement in diet resulted in a higher intake of protein and dietary fiber than the control group. The subjects who only received nutritional education and diet control decreased dietary fiber intake significantly after 5 weeks, but those who ate BGW during the test period showed increased dietary fiber intake. This finding is consistent with the previous study involving in dietary fiber of BGW. ${ }^{15,16}$ It indicates that, as a rich dietary fiber resource, BGW could play a beneficial role in human diet.

The improvement of dietary intake and the possible synergistic effects of BGW components should promote the hypoglycemic effect of BGW, which was confirmed in our subsequent experiments. HbAlc correlates with the average glucose more than GA but underestimates it, while GA reflects the glycemic variability more directly, so GA can serve as an indicator for hyperglycemia/hypoglycemia excursion. ${ }^{24}$ Recent studies suggested that GA is a better marker for glycemic control, especially for the evaluation of shorter term glycemic control in the general population with diabetes. In the present study, although blood glucose and $\mathrm{HbA} 1 \mathrm{c}$ were not significantly different between BGW and control groups, GA levels were affected significantly by BGW treatment during a 5-week shorter term intervention. GA levels in both groups were significantly decreased after the 5-week intervention compared with baseline, but the analysis of the change from baseline indicated that BGW treatment showed a much more efficient capacity to lower the GA level than diet control only. The subsequent subgroup analysis showed that the decreased GA level induced by BGW was effective when its intake was higher than $69 \mathrm{~g} / \mathrm{d}$. It means BGW intake may improve hyperglycemia and alleviate T2DM development.

Promotion of insulin secretion and/or inhibition of insulin resistance are the major mechanisms of glycemic control. Ingestion of whole-kernel rye food has been reported to reduce postprandial insulin, glucose-dependent insulinotropic polypeptide, and glucagon-like peptide 1 responses in healthy persons, compared with white wheat bread ingestion, but it did not decrease blood glucose obviously. ${ }^{17}$ Oat intake as staple food also decreased the insulin resistance HOMA-IR value when the dose reached $100 \mathrm{~g} / \mathrm{d}$ per person. ${ }^{19}$ However, the insulin concentration and HOMA-IR value were not affected significantly by BGW treatment after the 5-week intervention. It might be due to the fact that the daily intake of BGW was not sufficient enough or the duration of intervention was not long enough to show the benefits on $\beta$ cell function.

Beside of the insulin resistance and impaired insulin secretion, the activation of innate immunity provides a new model for the explanation of T2DM pathogenesis and MetS, which might result in new therapeutic approaches for the management of T2DM. There is increasing evidence that a chronic low-grade inflammation is closely involved in the pathogenesis of T2DM and its complications, because inflammation is an active part of the innate immune system. Elevated circulating inflammatory markers such as hsCRP, TNF- $\alpha$ and IL- 6 are strong predictors of T2DM development. ${ }^{25}$ In the present study, hs-CRP was not affected by $\mathrm{BGW}$ treatment during the test period, but $\mathrm{BGW}$ prevented the increase in TNF- $\alpha$ and IL-6 induced by T2DM. As the significant differences between the groups show, the subjects on only diet control showed significant increase in the TNF- $\alpha$ level after the 5-week test period, but subjects who consumed BGW did not. The difference in the IL-6 level between the 2 groups was also observed, but this effect was only attributed to the higher intake of BGW at a dose higher than $69 \mathrm{~g} / \mathrm{d}$. These findings indicate that the hypoglycemic effect of BGW is associated with its anti-inflammatory properties. The mechanism of T2DM alleviation by BGW might be similar to several anti-diabetic drugs (aspirin and thiazolidinediones), which lower both the inflammatory response and glycemia, so as to possibly decrease the T2DM risk.

The improvement of glycemia in response to the BGW treatment in T2DM subjects is closely associated with bioactive compounds of BGW. On one hand, protein and dietary fibers are mainly responsible for the hypoglycemic activity of BGW. Published data suggest that anthocyanins may lower blood glucose by improving insulin resistance, protecting $\beta$ cells, increasing secretion of insulin and reducing digestion of sugars in the small intestine. ${ }^{13} \mathrm{BGW}$ plays a role in lowering GA or glycemia by inhibiting glucose absorption in the intestine. On the other hand, protein and dietary fibers are mainly responsible for the hypoglycemic activity of BGW. BGW whole meal has higher protein content (17.71\%) than common wheat. Total essential amino acid contents, amino acid composition, and protein digestibility of BGW are relatively better than that of common wheat. ${ }^{16}$ Polysaccharides being an essential part of the dietary fiber composite are investigated in BGW. The fraction of polysaccharide in BGW is composed mainly of arabinose (36.53\%) and xylose $(61.31 \%) .{ }^{15}$ In addition, the antioxidant activity of phenolics might be related to the influence of BGW on glycemia and inflammation. The low-grade inflammation is called 
cytokine-induced acute-phase response, and hyperglycemiainduced cytokine production is mediated by reactive oxygen species, supporting the theory that the influence of BGW on the inflammatory profile might be associated with the antioxidant properties of its anthocyanins. Many in vitro and in vivo studies also reveal an array of mechanisms through which anthocyanins could prevent or reverse T2DM-related pathologies, including the promotion of antioxidant and antiinflammatory activities, improvement in insulin resistance, and hypolipidemic and hypoglycemic actions. ${ }^{12}$ The antidiabetic effect of anthocyanins are primarily related to their antioxidant properties, but enzymatic inhibition and other pathways may also be relevant, ${ }^{13}$ which is similar to the findings of black rice or purple corn. ${ }^{26,27}$

In conclusion, BGW intake $(>69 \mathrm{~g} / \mathrm{d})$ for 5 weeks decreased GA level, and prevented the increase in TNF- $\alpha$ and IL-6 levels compared with the control group, in which the subjects only received nutritional education and diet control. Consequently, the partial substitution of BGW for refined wheat or rice is an available option to alleviate or prevent hyperglycemia and inflammation in T2DM patients or in individuals at high risk of developing T2DM. The hypothesis for the research is thus accepted. Further studies could probably focus on larger sample sizes or longer durations to illustrate the influence of BGW on risk of T2DM or MetS.

\section{Acknowledgments}

We extend our appreciation to all technicians and the work group from Peking Union Medical College Hospital and Pinggu Hospital of Traditional Chinese Medicine for their help in dietary intake data collection, clinical data collection and laboratory analysis. This study was supported by the Agricultural Science and Technology Innovation Program of Chinese Academy of Agricultural Sciences on Minor Grain Nutrition Function.

\section{Disclosure}

The authors report no conflicts of interest in this work.

\section{References}

1. Yang W, Lu J, Weng J, et al; China National Diabetes and Metabolic Disorders Study Group. Prevalence of diabetes among men and women in China. N Engl J Med. 2010;362(12):1090-1101.

2. Boucher JL, Evert A, Daly A, et al; Merican Dietetics Association. American Dietetic Association revised standards of practice and standards of professional performance for registered Dietitians (generalist, specialty, and advanced) in diabetes care. J Am Diet Assoc. 2011; 111(1):156-166.

3. Franz MJ, Powers MA, Leontos C, et al. The evidence for medical nutrition therapy for type 1 and type 2 diabetes in adults. $J$ Am Diet Assoc. 2010;110(2):1852-1889.
4. Okarter N, Liu RH. Health benefits of whole grain phytochemicals Crit Rev Food Sci Nutr. 2010;50(3):193-208.

5. Sun SC, Yuan WY, Liu SX, et al. A study on the production of tetrageneric hybrids crossed between Triticum, agropyron, haynaldia, secale, and their morphology and cytology. Chin J Genet. 1996;23:131-53. Chinese.

6. Sun SC, Sun Y, Yuan WY, Yan WZ, Pei ZY, Zhang MR. Breeding and qualitative analysis for black wheat 76 of superior quality. Acta Agron Sin. 1999;25:51-54. Chinese.

7. Bai YF, Li WD, Sun SC, Sun Y, Pei ZY, Corke H. Evaluation on some quality characteristics of black-grained wheat 76. J Chinese Cereal Oil Assoc. 2000;15:6-9. Chinese.

8. Bai YF, Hou BY, Sun SC, Sun Y, Pei ZY, Yan GY. Genetic differences of gliadin profiles of black-grained wheat germplasm resources. J Triticeae Crop. 2002;22:22-25. Chinese.

9. Li WD, Sun SC, Ren GX. Study on characteristic of black wheat and its exploitation and utilization. China Condiment. 2004;1:9-11. Chinese

10. Sun Q, Sun B, Wang J. Characters of seed pigment of black kernel wheat. Seed. 2004;23:19-23. Chinese.

11. De Pascual-Teresa S, Sanchez-Ballesta MT. Anthocyanins: from plant to health. Phytochem Rev. 2008;7:281-299.

12. Guo H, Ling W. The update of anthocyanins on obesity and type 2 diabetes: experimental evidence and clinical perspectives. Rev Endocr Metab Disord. 2015;16(1):1-13.

13. Sancho RAS, Pastore GM. Evaluation of the effects of anthocyanins in type 2 diabetes. Food Res Int. 2012;46(1):378-386.

14. Li W, Shan F, Sun S, Corke H, Beta T. Free radical scavenging properties and phenolic content of Chinese black-brained wheat. J Agric Food Chem. 2005;53(22):8533-8536.

15. Sun Y, Cui SW, Gu X, Zhang J. Isolation and structural characterization of water unextractable arabinoxylans from Chinese black-grained wheat bran. Carbohyd Polym. 2011;85(3):615-621.

16. Li W, Beta T, Sun S, Corke H. Protein characteristics of Chinese blackgrained wheat. Food Chem. 2006;98:463-472.

17. Juntunen KS, Laaksonen DE, Autio K, et al. Structural differences between rye and wheat breads but not total fiber content may explain the lower postprandial insulin response to rye bread. Am J Clin Nutr. 2003;78(5):957-964.

18. Jensen MK, Koh-BanerjeeP, Franz M, Sampson L, Grønbæk M, RimmEB. Whole grains, bran, and germ in relation to homocysteine and markers of glycemic control, lipids, and inflammation. Am J Clin Nutr. 2006; 83(2):275-283.

19. Ma X, Gu J, Zhang Z, et al. Effects of avena nuda L. on metabolic control and cardiovascular disease risk among Chinese patients with diabetes and meeting metabolic syndrome criteria: secondary analysis of a randomized clinical trial. Eur J Clin Nutr. 2013;67(12):1291-1297.

20. Zhang G, Pan A, Zong G, et al. Substituting white rice with brown rice for 16 weeks does not substantially affect metabolic risk factors in middle-aged Chinese men and women with diabetes or a high risk for diabetes. $J$ Nutr. 2011;141(9):1685-1690.

21. Qiu J, Liu Y, Yue Y, Qin Y, Li Z. Dietary tartary buckwheat intake attenuates insulin resistance and improves lipid profiles in patients with type 2 diabetes: a randomized controlled trial. Nutr Res. 2016;36(12): $1392-1401$.

22. Wen D, Zhou XL, Li JJ, et al. Plasma concentrations of interleukin-6, C-reactive protein, tumor necrosis factor- $\alpha$ and matrix metalloproteinase- 9 in aortic dissection. Clinica Chimica Acta. 2012;413(1-2): 198-202.

23. Li JJ, Wang HR, Huang CX, Xue JL, Li GS. Enhanced inflammatory response of blood monocytes to C-reactive protein in patients with unstable angina. Clin Chim Acta. 2005;352(1-2):127-133.

24. Hayashi A, Takano K, Masaki T, Yoshino S, Ogawa A, Shichiri M. Distinct biomarker roles for HbAlc and glycated albumin in patients with type 2 diabetes on hemodialysis. J Diabetes Complications. 2016; 30(8):1494-1499. 
25. Pickup JC. Inflammation and activated innate immunity in the pathogenesis of type 2 diabetes. Diabetes Care. 2004;27(3):813-823.

26. Guo H, Ling W, Wang Q, et al. Effect of anthocyanin rich extract from black rice (Oryza sativa L. indica) on hyperlipidemia and insulin resistance in fructose-fed rats. Plant Food Hum Nutr. 2007;62(1):1-6.
27. Tsuda T, Horio F, Uchida K, Aoki H, Osawa T. Dietary cyanidin 3-O$\beta$-D-glucoside-rich purple corn color prevents obesity and ameliorates hyperglycaemia in mice. $J$ Nutr. 2003;133(7):2125-2130.

\section{Publish your work in this journal}

Therapeutics and Clinical Risk Management is an international, peerreviewed journal of clinical therapeutics and risk management, focusing on concise rapid reporting of clinical studies in all therapeutic areas, outcomes, safety, and programs for the effective, safe, and sustained use of medicines. This journal is indexed on PubMed Central, CAS,
EMBase, Scopus and the Elsevier Bibliographic databases. The manuscript management system is completely online and includes a very quick and fair peer-review system, which is all easy to use. Visit http://www.dovepress.com/testimonials.php to read real quotes from published authors.

Submit your manuscript here: http://www.dovepress.com/therapeutics-and-clinical-risk-management-journal 